FACTA UNIVERSITATIS (NIŠ)

Ser. Math. Inform. Vol. 33, No 2 (2018), 231-254

https://doi.org/10.22190/FUMI1802231P

\title{
VECTOR BUNDLES AND PARACONTACT FINSLER STRUCTURES
}

\author{
Esmaeil Peyghan and Esa Sharahi
}

\begin{abstract}
Almost paracontact and normal almost paracontact Finsler structures on a vector bundle are defined. Finding some conditions, integrability of these structures is studied. Moreover, we define paracontact metric, para- Sasakian and K-paracontact Finsler structures and study some properties of these structures. For a $K$-paracontact Finsler structure, we find vertical and horizontal flag curvatures. Then, defining the vertical $\varphi$-flag curvature, we prove that every locally symmetric para-Sasakian Finsler structure has a negative vertical $\varphi$-flag curvature. Finally, we define the horizontal and vertical Ricci tensors of a para-Sasakian Finsler structure and study some curvature properties of them.

Keywords: Finsler structure, paracontact structure, Sasakian structure, symmetry, vector bundle.
\end{abstract}

\section{Introduction}

Contact geometry has a very close relationship with physical concepts. This geometry was introduced by Sophus Lie in his works on PDEs. Contact theory is in contrast with foliation theory. In contact theory, the investigators try to study a distribution which is no longer integrable (even locally). This does not occur for any one-dimensional distribution, but in upper-dimensional distributions we can find such structures whose vector fields are not tangent to any submanifold of the main manifold.

If a notion can be investigated in the case of contact structures, it can be studied for paracontact structures as well. These structures were first introduced by Sato [11]. Then Sasaki focused on some interesting concepts of these structures when he studied as for contact structures $[9,10]$. Recently, many mathematician such as Bejan, Calvaruso, Druţă-Romaniuc, Ivanov, Kaneyuki, Cappelletti-Montano and Zamakovoy studied interesting properties of these structures $[1,2,3,4,7,8,17,18]$.

Received October 31, 2017; accepted December 12, 2017 2010 Mathematics Subject Classification. 53D15, 53C05 
The notion of vector bundle is one of many important geometric objects that have interesting applications in physics $[15,16]$. On vector bundles, Sinha, Prasad and Yadav defined some structures similar to the almost contact (paracontact) structures $[12,13,14]$. But the definitions presented by them are not well-defined (see 4.1) and cannot be realized in practical situations. Recently, Yalınıs and Calışkan introduced and studied some concepts about the contact structure on vector bundles based on the same definitions [19]. These incorrect definitions led to some bugs in their study (see 4.1, 4.3, 4.2). After studying and modifying the definitions, we submitted this paper to arxiv.org (see arXiv:1302.0647) in 2013. But in 2014, Kazan and Karadağ (without considering our paper) submitted and published a paper with similar results on paracontact structures on vector bundles. Also, their study was based on incorrect definitions (see [5]). Moreover, their study led to some pitfalls in numerous results and discussions. We mention these mistakes as remarks in the current text.

In this paper, we define almost paracontact Finsler structures and normal almost paracontact Finsler structures on a vector bundle $E$ and introduce some conditions for the integrability (normality) of these structures. We provide some equivalent conditions for the normality of an almost paracontact Finsler structure. Then, using a pseudo-metric $G$ on $E$, similarly to [17], we consider the following compatibility condition for this structure:

$$
G(\phi X, \phi Y)=-G(X, Y)+\eta(X) \eta(Y) .
$$

We also define the paracontact metric Finsler structure, para-Sasakian Finsler structure and K-paracontact Finsler structure. We find some conditions under which a paracontact metric Finsler structure is a K-paracontact structure. Then we get conditions under which a paracontact metric Finsler structure on a vector bundle $E$ reduces to a K-paracontact Finsler structure. For a K-paracontact Finsler structure on a vector bundle $E$, we find vertical and horizontal flag curvatures. We define the vertical $\phi$-flag curvature and prove that every locally symmetric para-Sasakian Finsler structure has a vertical $\phi$-flag curvature $-\frac{1}{4}$.

Finally, we define horizontal and vertical Ricci tensors of a para-Sasakian Finsler manifold and study some of their curvature properties.

\section{Preliminaries}

Let $E(M)=(E, \pi, M)$ be a vector bundle with an $(n+m)$-dimensional total space $E$, $n$-dimensional base space $M$ and the projection map $\pi$, such that $\pi: E \rightarrow M$, $u \in E \rightarrow \pi(u)=x \in M$ where $u=(x, y)$ and $y=\pi^{-1}(x)$ is the fibre of $E(M)$ over $x$. We denote by $V_{u} E$ the local fibre of the vertical bundle $V E$ at $u \in E$ and by $H_{u} E$ the complementary space of $V_{u} E$ in the tangent space $T_{u} E$ at $u$ to the total space $E$. Thus we have

$$
T_{u} E=H_{u} E \oplus V_{u} E
$$


A nonlinear connection $N$ on the total space $E$ of $E(M)$ is a differentiable distribution $H: E \rightarrow T_{u} E, u \in E \rightarrow H_{u} \subset T_{u} E$ with the property (2.1) (see [6]).

We denote by $\left(x^{i}, y^{a}\right), i=1, \ldots, n, a=1, \ldots, m$, the canonical coordinates of a point $u \in E$. Then $\left\{\frac{\partial}{\partial x^{i}}, \frac{\partial}{\partial y^{a}}\right\}$ is the natural basis and $\left\{d x^{i}, d y^{a}\right\}$ is its dual basis on $E$. It is easy to see that $\left\{\frac{\delta}{\delta x^{i}}, \frac{\partial}{\partial y^{a}}\right\}$ is the basis on $E$ adapted to decomposition (2.1) and $\left\{d x^{i}, \delta y^{a}\right\}$ is its basis (co-basis), where

$$
\frac{\delta}{\delta x^{i}}=\frac{\partial}{\partial x^{i}}-N_{i}^{a} \frac{\partial}{\partial y^{a}}, \quad \delta y^{a}=d y^{a}+N_{i}^{a} d x^{i},
$$

and $N_{i}^{a}$ are the coefficients of a nonlinear connection $N$. Now, we consider the horizontal and the vertical projectors $h$ and $v$ of the nonlinear connection, which are determined by the direct decomposition (2.1). These projectors can be expressed with respect to the adapted basis as follows:

$$
h=\frac{\delta}{\delta x^{i}} \otimes d x^{i}, \quad v=\frac{\partial}{\partial y^{a}} \otimes \delta y^{a} .
$$

Using the above projectors, any vector field $X$ on $E$ can be uniquely written as $X=h X+v X$. In the following, we adopt the notations

$$
h X=X^{H}, \quad v X=X^{V}
$$

and we say $X^{H}$ and $X^{V}$ are horizontal and vertical components of $X$. Thus, any vector field $X$ on $E$ can be uniquely written in the form

$$
X=X^{H}+X^{V} .
$$

In the adapted basis, we have $X=X^{i}(x, y) \frac{\delta}{\delta x^{i}}+\bar{X}^{a}(x, y) \frac{\partial}{\partial y^{a}}$ and

$$
X^{H}=X^{i}(x, y) \frac{\delta}{\delta x^{i}}, \quad X^{V}=\bar{X}^{a}(x, y) \frac{\partial}{\partial y^{a}} .
$$

Now, let $\omega$ be a 1 -form on $E$. Then it can be uniquely written as $\omega=\omega^{H}+\omega^{V}$. In the adapted basis, we have $\omega=\omega_{i}(x, y) d x^{i}+\bar{\omega}_{a}(x, y) \delta y^{a}$ and

$$
\omega^{H}=\omega_{i}(x, y) d x^{i}, \quad \omega^{V}=\bar{\omega}_{a}(x, y) \delta y^{a} .
$$

A tensor field $T$ on the vector bundle $E$ is called a distinguished tensor field (briefly, a d-tensor) of type $\left(\begin{array}{cc}p & r \\ q & s\end{array}\right)$ if it has the following property

$$
\begin{aligned}
& T\left(\omega_{i_{1}}, \ldots, \omega_{i_{p}}, \omega_{a_{1}}, \ldots, \omega_{a_{r}}, X_{j_{1}}, \ldots, X_{j_{q}}, X_{b_{1}}, \ldots, X_{b_{s}}\right) \\
& \quad=T\left(\omega_{i_{1}}^{H}, \ldots, \omega_{i_{p}}^{H}, \omega_{a_{1}}^{V}, \ldots, \omega_{a_{r}}^{V}, X_{j_{1}}^{H}, \ldots, X_{j_{q}}^{H}, X_{b_{1}}^{V}, \ldots, X_{b_{s}}^{V}\right),
\end{aligned}
$$

where $\omega_{i_{k}}, \omega_{a_{l}},(k=1, \ldots, p, l=1, \ldots, r)$ are 1 -forms on $E$ and $X_{j_{v}}, X_{b_{w}},(v=$ $1, \ldots, q, w=1, \ldots, s)$ are vector fields on $E$. For instance, the components $X^{H}$ and 
$X^{V}$ from (2.2) of a vector field $X$ are d-vector fields. Also the components $\omega^{H}$ and $\omega^{V}$ of an 1 -form $\omega$, from $(2.3)$ are d-1-form fields. In the adapted basis $\left\{\frac{\delta}{\delta x^{i}}, \frac{\partial}{\partial y^{a}}\right\}$ and adapted co-basis $\left\{d x^{i}, \delta y^{a}\right\}, T$ is expressed by

$$
\begin{aligned}
T= & T_{j_{1}, \ldots, j_{q}, b_{1}, \ldots, b_{s}}^{i_{1}, \ldots, i_{p}, a_{1}, \ldots, a_{r}} \frac{\delta}{\delta x^{i_{1}}} \otimes \ldots \otimes \frac{\delta}{\delta x^{i_{p}}} \otimes \frac{\partial}{\partial y^{a_{1}}} \otimes \ldots \otimes \frac{\partial}{\partial y^{a_{r}}} \\
& \otimes d x^{j_{1}} \otimes \ldots \otimes d x^{j_{q}} \otimes \delta y^{b_{1}} \otimes \ldots \otimes \delta y^{b_{s}} .
\end{aligned}
$$

A linear connection $D$ on $E$ is called a distinguished connection (briefly, dconnection) if it preserves by parallelism the horizontal distribution, that is $D h=0$. Since $I d=h+v$, then $D h=0$ implies that $D v=0$. Thus a d-connection preserves by parallelism the vertical distribution. Therefore, we can write

$$
\begin{aligned}
D_{X} Y & =\left(D_{X} Y^{H}\right)^{H}+\left(D_{X} Y^{V}\right)^{V}, \\
D_{X} \omega & =\left(D_{X} \omega^{H}\right)^{H}+\left(D_{X} \omega^{V}\right)^{V},
\end{aligned}
$$

where $X, Y$ are vector fields on $E$ and $\omega$ is a 1-form on $E$.

A d-connection with respect to the adapted basis has the following form

$$
\left\{\begin{aligned}
D_{\frac{\delta}{\delta x^{i}}} \frac{\delta}{\delta x^{j}}=F_{i j}^{k} \frac{\delta}{\delta x^{k}}, & D_{\frac{\delta}{\delta x^{i}}} \frac{\partial}{\partial y^{b}}=\bar{F}_{i b}^{c} \frac{\partial}{\partial y^{c}}, \\
D_{\frac{\partial}{\partial y^{a}}} \frac{\delta}{\delta x^{j}}=C_{a j}^{k} \frac{\delta}{\delta x^{k}}, & D_{\frac{\partial}{\partial y^{a}} \frac{\partial}{\partial y^{b}}}=\bar{C}_{a b}^{c} \frac{\partial}{\partial y^{c}} .
\end{aligned}\right.
$$

For this connection, there is an associated pair of operators in the algebra of d-tensor fields. For any vector field $X$ on $E$, set

$$
D_{X}^{H} Y=D_{X^{H}} Y, \quad D_{X}^{V} Y=D_{X^{V}} Y \quad D_{X}^{H} f=X^{H}(f), \quad D_{X}^{V} f=X^{V}(f),
$$

where $Y$ is a vector field and $f$ is a smooth function on $E$. We call $D^{H}\left(D^{V}\right)$ the operator of $h$-covariant ( $v$-covariant) derivation. If $\omega$ is a 1 -form on $E$, we define

$$
\begin{aligned}
& \left(D_{X}^{H} \omega\right) Y=X^{H}(\omega(Y))-\omega\left(D_{X}^{H} Y\right), \\
& \left(D_{X}^{V} \omega\right) Y=X^{V}(\omega(Y))-\omega\left(D_{X}^{V} Y\right),
\end{aligned}
$$

for any vector fields $X, Y$ on $E$.

Now, we consider the pseudo-metric structure $G$ on $E$ which is symmetric and non-degenerate as $G=G^{H}+G^{V}$, where $G^{H}(X, Y)=G\left(X^{H}, Y^{H}\right)$ is of type $\left(\begin{array}{ll}0 & 0 \\ 2 & 0\end{array}\right)$, symmetric and non-degenerate on $H_{u} E$ and $G^{V}(X, Y)=G\left(X^{V}, Y^{V}\right)$ is of type $\left(\begin{array}{cc}0 & 0 \\ 0 & 2\end{array}\right)$, symmetric and non-degenerate on $V_{u} E$. In the adapted basis, we can write

$$
G=g_{i j}(x, y) d x^{i} \otimes d x^{j}+h_{a b}(x, y) \delta y^{i} \otimes \delta y^{j} .
$$

A d-connection $D$ on $E$ is called a metrical d-connection with respect to $G$ if $D_{X} G=0$ holds for every vector field $X$ on $E$. 
For a d-connection $D$, we consider the torsion $T$ defined by

$$
T(X, Y)=D_{X} Y-D_{Y} X-[X, Y], \quad \forall X, Y \in \chi(E),
$$

where $\chi(E)$ is the set of all vector fields on $E$. The torsion of a d-connection $D$ on $E$ is completely determined by the following five tensor fields:

$$
\begin{aligned}
& T^{H}\left(X^{H}, Y^{H}\right)=D_{X}^{H} Y^{H}-D_{Y}^{H} X^{H}-\left[X^{H}, Y^{H}\right]^{H}, \\
& T^{V}\left(X^{H}, Y^{H}\right)=-\left[X^{H}, Y^{H}\right]^{V}, \\
& T^{H}\left(X^{H}, Y^{V}\right)=-D_{Y}^{V} X^{H}-\left[X^{H}, Y^{V}\right]^{H}, \\
& T^{V}\left(X^{H}, Y^{V}\right)=D_{X}^{H} Y^{V}-\left[X^{H}, Y^{V}\right]^{V} \\
& T^{V}\left(X^{V}, Y^{V}\right)=D_{X}^{V} Y^{V}-D_{Y}^{V} X^{V}-\left[X^{V}, Y^{V}\right]^{V},
\end{aligned}
$$

which are called $(h) h$-torsion, $(v) h$-torsion, $(h) h v$-torsion, $(v) h v$-torsion and $(v) v$ torsion, respectively. A d-connection $D$ is said to be symmetric if the $(h) h$-torsion and $(v) v$-torsion vanish. In this paper, we use the symmetric metrical d-connection and we call it Finsler connection. It is easy to see that the following relations hold for the Finsler connection

$$
\begin{gathered}
2 G\left(D_{X}^{H} Y^{H}, Z^{H}\right)=X^{H} G\left(Y^{H}, Z^{H}\right)+Y^{H} G\left(X^{H}, Z^{H}\right)-Z^{H} G\left(X^{H}, Y^{H}\right) \\
+G\left(\left[X^{H}, Y^{H}\right], Z^{H}\right)-G\left(\left[X^{H}, Z^{H}\right], Y^{H}\right)-G\left(\left[Y^{H}, Z^{H}\right], X^{H}\right) \\
2 G\left(D_{X}^{V} Y^{V}, Z^{V}\right)=X^{V} G\left(Y^{V}, Z^{V}\right)+Y^{V} G\left(X^{V}, Z^{V}\right)-Z^{V} G\left(X^{V}, Y^{V}\right) \\
+G\left(\left[X^{V}, Y^{V}\right], Z^{V}\right)-G\left(\left[X^{V}, Z^{V}\right], Y^{V}\right)-G\left(\left[Y^{V}, Z^{V}\right], X^{V}\right) .
\end{gathered}
$$

Finally, we consider the curvature of a Finsler connection $D$ as follows

$$
R(X, Y) Z=D_{X} D_{Y} Z-D_{Y} D_{X} Z-D_{[X, Y]} Z, \quad \forall X, Y, Z \in \chi(E) .
$$

As $D$ preserves by parallelism the horizontal and vertical distributions, from the above equation, we see that the operator $R(X, Y)$ carries horizontal vector fields into horizontal vector fields and vertical vector fields into verticals. Consequently, we have the following

$$
R(X, Y) Z=\left(R(X, Y) Z^{H}\right)^{H}+\left(R(X, Y) Z^{V}\right)^{V} \quad \forall X, Y, Z \in \chi(E) .
$$

Since $R(X, Y)$ is skew symmetric with respect to $X$ and $Y$, then the curvature of a Finsler connection $D$ on $E$ is completely determined by the following six tensor fields

$$
\left\{\begin{array}{c}
R\left(X^{H}, Y^{H}\right) Z^{H}=D_{X}^{H} D_{Y}^{H} Z^{H}-D_{Y}^{H} D_{X}^{H} Z^{H}-D_{\left[X^{H}, Y^{H}\right]} Z^{H} \\
R\left(X^{H}, Y^{H}\right) Z^{V}=D_{X}^{H} D_{Y}^{H} Z^{V}-D_{Y}^{H} D_{X}^{H} Z^{V}-D_{\left[X^{H}, Y^{H}\right]} Z^{V} \\
R\left(X^{V}, Y^{H}\right) Z^{H}=D_{X}^{V} D_{Y}^{H} Z^{H}-D_{Y}^{H} D_{X}^{V} Z^{H}-D_{\left[X^{V}, Y^{H}\right]} Z^{H} \\
R\left(X^{V}, Y^{H}\right) Z^{V}=D_{X}^{V} D_{Y}^{H} Z^{V}-D_{Y}^{H} D_{X}^{V} Z^{V}-D_{\left[X^{V}, Y^{H}\right]} Z^{V} \\
R\left(X^{V}, Y^{V}\right) Z^{H}=D_{X}^{V} D_{Y}^{V} Z^{H}-D_{Y}^{V} D_{X}^{V} Z^{H}-D_{\left[X^{V}, Y^{V}\right]} Z^{H} \\
R\left(X^{V}, Y^{V}\right) Z^{V}=D_{X}^{V} D_{Y}^{V} Z^{V}-D_{Y}^{V} D_{X}^{V} Z^{V}-D_{\left[X^{V}, Y^{V}\right]} Z^{V}
\end{array}\right.
$$

In the sequel, the restriction of the tensor field $R$ to the horizontal (respectively vertical) distribution will be called horizontal (respectively vertical) curvature of $D$. 


\section{Almost Paracontact Finsler Structure}

We consider a tensor field $\phi$, a 1-form $\eta$ and a vector field $\xi$ on $E$, given by:

$$
\begin{gathered}
\phi=\phi_{j}^{i}(x, y) \frac{\delta}{\delta x^{i}} \otimes d x^{j}+\bar{\phi}_{b}^{a}(x, y) \frac{\partial}{\partial y^{a}} \otimes \delta y^{b}, \\
\eta=\eta_{i}(x, y) d x^{i}+\bar{\eta}_{a}(x, y) \delta y^{a}, \quad \xi=\xi^{i}(x, y) \frac{\delta}{\delta x^{i}}+\bar{\xi}^{a}(x, y) \frac{\partial}{\partial y^{a}} .
\end{gathered}
$$

Definition 3.1. Suppose that $\phi, \eta$ and $\xi$ are given by (3.1) and (3.2) on $E$ such that

$$
\phi^{2}=I-\eta^{H} \otimes \xi^{H}-\eta^{V} \otimes \xi^{V}, \quad \eta^{H}\left(\xi^{H}\right)=\eta^{V}\left(\xi^{V}\right)=1,
$$

where

$$
\eta^{H}=\eta_{i}(x, y) d x^{i}, \quad \eta^{V}=\bar{\eta}_{a}(x, y) \delta y^{a}, \quad \xi^{H}=\xi^{i}(x, y) \frac{\delta}{\delta x^{i}}, \quad \xi^{V}=\bar{\xi}^{a}(x, y) \frac{\partial}{\partial y^{a}} .
$$

Then $(\phi, \eta, \xi)$ is called an almost paracontact Finsler structure on $E$ and $E$ is called an almost paracontact Finsler vector bundle.

Now, we are going to consider some properties of an almost paracontact Finsler structure. First, we prove the following.

Theorem 3.1. Suppose that $E$ has an almost paracontact Finsler structure, then the following holds

$$
\phi\left(\xi^{H}\right)=\phi\left(\xi^{V}\right)=0, \quad \eta^{H} \circ \phi=\eta^{V} \circ \phi=0 .
$$

Proof. By (3.3) and $\eta^{V}\left(\xi^{H}\right)=0$, we have

$$
\phi^{2}\left(\xi^{H}\right)=\xi^{H}-\eta^{H}\left(\xi^{H}\right) \xi^{H}=0 .
$$

Then $\phi\left(\xi^{H}\right)=0$ or $\phi\left(\xi^{H}\right)$ is a nontrivial eigenvector of $\phi$ corresponding to the eigenvalue 0. Since $\phi\left(\xi^{H}\right) \in H E$, then $\eta^{V}\left(\phi\left(\xi^{H}\right)\right)=0$. Using (3.3), we obtain

$$
0=\phi^{2}\left(\phi\left(\xi^{H}\right)\right)=\phi\left(\xi^{H}\right)-\eta^{H}\left(\phi\left(\xi^{H}\right)\right) \xi^{H} \quad \text { or } \quad \phi\left(\xi^{H}\right)=\eta^{H}\left(\phi\left(\xi^{H}\right)\right) \xi^{H} .
$$

Now, if $\phi\left(\xi^{H}\right)$ is nontrivial eigenvector of the eigenvalue 0 , then $\eta^{H}\left(\phi\left(\xi^{H}\right)\right) \neq 0$. Thus we have

$$
0=\phi^{2}\left(\xi^{H}\right)=\eta^{H}\left(\phi\left(\xi^{H}\right)\right) \phi\left(\xi^{H}\right)=\left(\eta^{H}\left(\phi\left(\xi^{H}\right)\right)\right)^{2} \xi^{H} \neq 0,
$$

which is a contradiction. Therefore $\phi\left(\xi^{H}\right)=0$. Similarly, we get $\phi\left(\xi^{V}\right)=0$.

On the other hand, since $\phi\left(\xi^{H}\right)=0$ then we get

$$
\begin{aligned}
\eta^{H}(\phi(X)) \xi^{H} & =\eta^{H}\left(\phi\left(X^{H}\right)\right) \xi^{H}=\phi\left(X^{H}\right)-\phi^{3}\left(X^{H}\right) \\
& =\phi\left(X^{H}\right)-\phi\left(X^{H}\right)+\phi\left(\eta^{H}\left(X^{H}\right) \xi^{H}\right)=0,
\end{aligned}
$$

for any $X \in \chi(E)$. Hence $\eta^{H} \circ \phi=0$. Similarly, we have $\eta^{V} \circ \phi=0$. 
Remark 3.1. Let us put

$$
\phi^{H}=\phi_{j}^{i}(x, y) \frac{\delta}{\delta x^{i}} \otimes d x^{j} \quad \text { and } \quad \phi^{V}=\bar{\phi}_{b}^{a} \frac{\partial}{\partial y^{a}} \otimes \delta y^{b} .
$$

Then by Theorem 3.1, we deduce that $\left(\phi^{H}, \eta^{H}, \xi^{H}\right)$ and $\left(\phi^{V}, \eta^{V}, \xi^{V}\right)$ are almost paracontact structures on sub-bundles $H E$ and $V E$, respectively.

Proposition 3.1. Let $E$ be endowed with an almost paracontact Finsler structure $(\phi, \eta, \xi)$. Then $\operatorname{rank} \phi=(\operatorname{dim} E)-2$.

Proof. It is sufficient to show that $\operatorname{ker} \phi=<\xi^{H}>\oplus<\xi^{V}>$. Since $\phi \xi^{H}=\phi \xi^{V}=0$, then we have $\left\langle\xi^{H}>\oplus\left\langle\xi^{V}>\subseteq \operatorname{ker} \phi\right.\right.$. Now, let $\bar{\xi} \in \operatorname{ker} \phi$. Then $\phi \bar{\xi}=0$ and (3.3) give us

$$
\bar{\xi}=\eta^{H}(\bar{\xi}) \xi^{H}+\eta^{V}(\bar{\xi}) \eta^{V} \in<\xi^{H}>\oplus<\xi^{V}>,
$$

i.e., $\operatorname{ker} \phi \subseteq<\xi^{H}>\oplus<\xi^{V}>$. Thus $\operatorname{ker} \phi=<\xi^{H}>\oplus<\xi^{V}>$.

We say that an almost paracontact Finsler structure $(\phi, \eta, \xi)$ on the vector bundle $E$ is normal, if the following holds

$$
N^{(1)}(X, Y)=N_{\phi}(X, Y)-d \eta^{H}(X, Y) \xi^{H}-d \eta^{V}(X, Y) \xi^{V}=0,
$$

where $X, Y$ are vector fields on $E$.

Now, we are going to give some equivalent conditions for the normality of structure $(\phi, \eta, \xi)$. For this reason, we introduce three tensors $N^{(2)}, N^{(3)}$ and $N^{(4)}$ and show that the vanishing of $N^{(1)}$ implies the vanishing of these tensors. First, we define the tensor $N^{(2)}$ on $T_{u} E$ as follows

$$
\begin{aligned}
N^{(2)}\left(X^{H}, Y^{H}\right)= & \left(£_{\phi X}^{H} \eta^{H}\right)\left(Y^{H}\right)-\left(£_{\phi Y}^{H} \eta^{H}\right)\left(X^{H}\right), \\
N^{(2)}\left(X^{V}, Y^{V}\right)= & \left(£_{\phi X}^{V} \eta^{V}\right)\left(Y^{V}\right)-\left(£_{\phi Y}^{V} \eta^{V}\right)\left(X^{V}\right), \\
N^{(2)}\left(X^{V}, Y^{H}\right)= & \left(£_{\phi X}^{V} \eta^{H}\right)\left(Y^{H}\right)+\left(£_{\phi X}^{V} \eta^{V}\right)\left(Y^{H}\right) \\
& -\left(£_{\phi Y}^{H} \eta^{H}\right)\left(X^{V}\right)-\left(£_{\phi Y}^{H} \eta^{V}\right)\left(X^{V}\right) .
\end{aligned}
$$

To define $N^{(3)}$ and $N^{(4)}$, we consider the following cases:

Case 1: For $X^{H}, \xi^{H} \in H_{u} E$, we define

$$
N^{(3)}\left(X^{H}\right)=\left(£_{\xi}^{H} \phi\right)\left(X^{H}\right), \quad N^{(4)}\left(X^{H}\right)=\left(£_{\xi}^{H} \eta^{H}\right)\left(X^{H}\right) .
$$

Case 2: For $X^{V}, \xi^{V} \in V_{u} E$, we define

$$
N^{(3)}\left(X^{V}\right)=\left(£_{\xi}^{V} \phi\right)\left(X^{V}\right), \quad N^{(4)}\left(X^{V}\right)=\left(£_{\xi}^{V} \eta^{V}\right)\left(X^{V}\right) .
$$

Case 3: For $X^{H} \in H_{u} E$ and $\xi^{V} \in V_{u} E$, we define

$$
N^{(3)}\left(X^{H}\right)=\left(£_{\xi}^{V} \phi\right)\left(X^{H}\right), \quad N^{(4)}\left(X^{H}\right)=\left(£_{\xi}^{V} \eta^{H}\right)\left(X^{H}\right) .
$$

Case 4: For $X^{V} \in V_{u} E$ and $\xi^{H} \in H_{u} E$, we define

$$
N^{(3)}\left(X^{V}\right)=\left(£_{\xi}^{H} \phi\right)\left(X^{V}\right), \quad N^{(4)}\left(X^{V}\right)=\left(£_{\xi}^{H} \eta^{V}\right)\left(X^{V}\right) .
$$


Theorem 3.2. For any almost paracontact Finsler structure $(\phi, \eta, \xi)$ the vanishing of $N^{(1)}$ implies the vanishing of $N^{(2)}, N^{(3)}$ and $N^{(4)}$.

Proof. If $N^{(1)}=0$, then for $X^{H}$ and $\xi^{H}$ we have

$$
\begin{aligned}
0 & =N^{(1)}\left(X^{H}, \xi^{H}\right) \\
& =\phi^{2}\left[X^{H}, \xi^{H}\right]+\left[\phi X^{H}, \phi \xi^{H}\right]-\phi\left[\phi X^{H}, \xi^{H}\right]-\phi\left[X^{H}, \phi \xi^{H}\right] \\
& -d \eta^{H}\left(X^{H}, \xi^{H}\right) \xi^{H}-d \eta^{V}\left(X^{H}, \xi^{H}\right) \xi^{V} \\
& =\phi^{2}\left[X^{H}, \xi^{H}\right]-\phi\left[\phi X^{H}, \xi^{H}\right]-d \eta^{H}\left(X^{H}, \xi^{H}\right) \xi^{H}-d \eta^{V}\left(X^{H}, \xi^{H}\right) \xi^{V} .
\end{aligned}
$$

Applying $\eta^{H}$ to (3.4), we obtain

$$
d \eta^{H}\left(X^{H}, \xi^{H}\right)=0,
$$

which gives

$$
\begin{aligned}
N^{(4)}\left(X^{H}\right)=\left(£_{\xi}^{H} \eta^{H}\right)\left(X^{H}\right) & =\xi^{H}\left(\eta^{H}\left(X^{H}\right)\right)-\eta^{H}\left[\xi^{H}, X^{H}\right] \\
& =-d \eta^{H}\left(X^{H}, \xi^{H}\right)=0 .
\end{aligned}
$$

Since $d \eta^{H}\left(X^{H}, \xi^{H}\right)=0$, then by (3.4) we have

$$
0=\phi^{2}\left[X^{H}, \xi^{H}\right]-\phi\left[\phi X^{H}, \xi^{H}\right]=\phi\left(\left(£_{\xi}^{H} \phi\right) X^{H}\right) .
$$

Similarly to (3.4), we obtain

$$
\begin{aligned}
& 0=\eta^{H}\left(N^{(1)}\left(\phi X^{H}, \xi^{H}\right)\right)=d \eta^{H}\left(\xi^{H}, \phi X^{H}\right), \\
& 0=\eta^{V}\left(N^{(1)}\left(\phi X^{H}, \xi^{H}\right)\right)=d \eta^{V}\left(\xi^{H}, \phi X^{H}\right),
\end{aligned}
$$

which imply that

$$
\eta^{H}\left(\left[\xi^{H}, \phi X^{H}\right]\right)=0, \quad \eta^{V}\left(\left[\xi^{H}, \phi X^{H}\right]\right)=0 .
$$

Applying $\phi$ to (3.4) and using the above equation, we have $\left(£_{\xi}^{H} \phi\right) X^{H}=0$, i.e., $N^{(3)}\left(X^{H}\right)=0$. Applying $\eta^{H}$ to the following

$$
\begin{aligned}
0= & N^{(1)}\left(\phi X^{H}, Y^{H}\right)=\left[X^{H}, \phi Y^{H}\right]-\eta^{H}\left(X^{H}\right)\left[\xi^{H}, \phi Y^{H}\right]+\phi Y^{H}\left(\eta^{H}\left(X^{H}\right)\right) \xi^{H} \\
& -\phi\left[X^{H}, Y^{H}\right]-\phi\left[\phi X^{H}, \phi Y^{H}\right]+\left[\phi X^{H}, Y^{H}\right]-\phi X^{H}\left(\eta^{H}\left(Y^{H}\right)\right) \xi^{H} \\
& +\eta^{H}\left(X^{H}\right) \phi\left[\xi^{H}, Y^{H}\right]+\eta^{V}\left[\phi X^{H}, Y^{H}\right] \xi^{V}
\end{aligned}
$$

and using $\eta^{H}\left(\left[\xi^{H}, \phi X^{H}\right]\right)=0$, we get

$$
\begin{aligned}
0 & =-\eta^{H}\left[\phi Y^{H}, X^{H}\right]+\phi Y^{H}\left(\eta^{H}\left(X^{H}\right)\right)+\eta^{H}\left[\phi X^{H}, Y^{H}\right]-\phi X^{H}\left(\eta^{H}\left(Y^{H}\right)\right) \\
& =\left(£_{\phi Y}^{H} \eta^{H}\right) X^{H}-\left(£_{\phi X}^{H} \eta^{H}\right) Y^{H} .
\end{aligned}
$$


Thus $N^{(2)}\left(X^{H}, Y^{H}\right)=0$. In a similar way, we can conclude the vanishing of $N^{(2)}$, $N^{(3)}$ and $N^{(4)}$ from the vanishing of $N^{(1)}$, when $X^{V}$ and $Y^{V}$ belong to $V_{u} E$. Now we prove the result when one of them belongs to $V_{u} E$ and the other belongs to $H_{u} E$.

Similarly to (3.4), the vanishing of $N^{(1)}$ implies that

$$
\begin{aligned}
0 & =N^{(1)}\left(X^{V}, \xi^{H}\right) \\
& =\phi^{2}\left[X^{V}, \xi^{H}\right]-\phi\left[\phi X^{V}, \xi^{H}\right]-d \eta^{H}\left(X^{V}, \xi^{H}\right) \xi^{H}-d \eta^{V}\left(X^{V}, \xi^{H}\right) \xi^{V} .
\end{aligned}
$$

Applying $\eta^{V}$ and $\eta^{H}$ to (3.5), we get

$$
d \eta^{V}\left(X^{V}, \xi^{H}\right)=0, \quad d \eta^{H}\left(X^{V}, \xi^{H}\right)=0
$$

But we have

$$
N^{(4)}\left(X^{V}\right)=\left(£_{\xi}^{H} \eta^{V}\right)\left(X^{V}\right)=\xi^{H}\left(\eta^{V}\left(X^{V}\right)\right)-\eta^{V}\left[\xi^{H}, X^{V}\right]=-d \eta^{V}\left(X^{V}, \xi^{H}\right) .
$$

Therefore the first part of (3.6) gives us $N^{(4)}\left(X^{V}\right)=0$. Using (3.5) and (3.6), we obtain

$$
\begin{aligned}
0=\phi\left(N^{(1)}\left(X^{V}, \xi^{H}\right)\right) & =\phi\left[X^{V}, \xi^{H}\right]-\left[\phi X^{V}, \xi^{H}\right] \\
& =\left(£_{\xi}^{H} \phi\right)\left(X^{V}\right) \\
& =N^{(3)}\left(X^{V}\right) .
\end{aligned}
$$

Therefore $N^{(3)}\left(X^{V}\right)=0$. In a similar way to (3.5), we obtain

$$
\begin{aligned}
& 0=\eta^{H}\left(N^{(1)}\left(\xi^{V}, \phi Y^{H}\right)\right)=-d \eta^{H}\left(\xi^{V}, \phi Y^{H}\right), \\
& 0=\eta^{V}\left(N^{(1)}\left(\xi^{V}, \phi Y^{H}\right)\right)=-d \eta^{V}\left(\xi^{V}, \phi Y^{H}\right)
\end{aligned}
$$

which gives us

$$
\eta^{H}\left[\xi^{V}, \phi Y^{H}\right]=0, \quad \eta^{V}\left[\xi^{V}, \phi Y^{H}\right]=0
$$

Using (3.7), we get

$$
\begin{aligned}
0= & \eta\left(N^{(1)}\left(\phi X^{V}, Y^{H}\right)\right) \\
= & \eta^{H}\left(\left[X^{V}, \phi Y^{H}\right]\right)+\eta^{V}\left(\left[X^{V}, \phi Y^{H}\right]\right)+\phi Y^{H}\left(\eta^{V}\left(X^{V}\right)\right)+\eta^{V}\left(\left[\phi X^{V}, Y^{H}\right]\right) \\
& \quad-\phi X^{V}\left(\eta^{H}\left(Y^{H}\right)\right)+\eta^{H}\left(\left[\phi X^{V}, Y^{H}\right]\right) \\
= & -N^{(2)}\left(X^{V}, Y^{H}\right)
\end{aligned}
$$

i.e., $N^{(2)}\left(X^{V}, Y^{H}\right)=0$. 


\section{Paracontact Finsler Structures}

A pseudo-metric structure $G$ on $E$ satisfying the conditions

$$
\begin{gathered}
G^{H}(\phi X, \phi Y)=-G^{H}(X, Y)+\eta^{H}(X) \eta^{H}(Y), \\
G^{V}(\phi X, \phi Y)=-G^{V}(X, Y)+\eta^{V}(X) \eta^{V}(Y),
\end{gathered}
$$

is said to be compatible with the structure $(\phi, \eta, \xi)$. In this case, the quadruplet $(\phi, \eta, \xi, G)$ is called an almost paracontact metric Finsler structure and $E$ is called an almost paracontact metric Finsler vector bundle. From (4.1) and (4.2) we deduce

$$
G(\phi X, \phi Y)=-G(X, Y)+\eta^{H}(X) \eta^{H}(Y)+\eta^{V}(X) \eta^{V}(Y) .
$$

By (4.1) and (4.2) we have

$$
G^{H}(X, \xi)=\eta^{H}(X), \quad G^{V}(X, \xi)=\eta^{V}(X),
$$

which gives us $G(X, \xi)=\eta(X)$. Using (4.1)-(4.3), one can also obtain

$$
G\left(X^{H}, \phi Y^{H}\right)=-G\left(\phi X^{H}, Y^{H}\right), \quad G\left(X^{V}, \phi Y^{V}\right)=-G\left(\phi X^{V}, Y^{V}\right) .
$$

Now, we define the fundamental 2 -form $\Phi$ by

$$
\Phi(X, Y)=G(X, \phi Y), \quad \forall X, Y \in \chi(E),
$$

which gives

$$
\begin{aligned}
& \Phi\left(X^{H}, Y^{H}\right)=G^{H}(X, \phi Y), \quad \Phi\left(X^{V}, Y^{V}\right)=G^{V}(X, \phi Y), \\
& \Phi\left(X^{V}, Y^{H}\right)=-\Phi\left(Y^{H}, X^{V}\right)=G\left(X^{V}, \phi Y^{H}\right)=0 .
\end{aligned}
$$

Remark 4.1. In [5, 12, 13, 14, 19], to define contact and paracontact Finsler structures the authors considered a tensor field $\phi$ of type $\left(\begin{array}{ll}1 & 1 \\ 1 & 1\end{array}\right)$. According to (2.4), $\phi$ has the following local expression

$$
\phi=\phi_{j b}^{i a} \frac{\delta}{\delta x^{i}} \otimes \frac{\partial}{\partial y^{a}} \otimes d x^{j} \otimes \delta y^{b} .
$$

Thus for $X=X^{k}(x, y) \frac{\delta}{\delta x^{k}}+\bar{X}^{c}(x, y) \frac{\partial}{\partial y^{c}}$ we have

$$
\phi(X)=\phi_{k b}^{i a} X^{k} \frac{\delta}{\delta x^{i}} \otimes \frac{\partial}{\partial y^{a}} \otimes \delta y^{b}+\phi_{j c}^{i a} \bar{X}^{c} \frac{\delta}{\delta x^{i}} \otimes d x^{j} \otimes \frac{\partial}{\partial y^{a}} .
$$

This shows that $\phi(X)$ is not a vector field on $E$ and so $G(\phi X, \phi Y)$ is not welldefined. Therefore, $\phi$ can not be a tensor field of type $\left(\begin{array}{ll}1 & 1 \\ 1 & 1\end{array}\right)$. Also, in the definition of contact and paracontact Finsler structures they considered the condition

$$
\eta^{H}\left(\xi^{H}\right)+\eta^{V}\left(\xi^{V}\right)=1
$$


and using it, they deduced $G^{H}(X, \xi)=\eta^{H}(X)$ from $G^{H}(\phi X, \phi Y)=-G^{H}(X, Y)+$ $\eta^{H}(X) \eta^{H}(Y)$ or $G^{H}(\phi X, \phi Y)=G^{H}(X, Y)-\eta^{H}(X) \eta^{H}(Y)$ (see (3.9) of [5] and (2.8) of [19]). But it is easy to see that this result is not true unless $\eta^{H}\left(\xi^{H}\right)=$ 1. In a similar way, we can deduce that the condition $\eta^{V}\left(\xi^{V}\right)=1$ is necessary. According to these reasons, definitions of contact and paracontact Finsler structures in [5, 12, 13, 14, 19] are not true mathematically. Moreover, the condition (4.5) breaks down the idea of inheritance properties by vertical and horizontal slices of a paracontact structure on a vector bundle (obviously this idea needs the condition $\eta^{H}\left(\xi^{H}\right)=\eta^{V}\left(\xi^{V}\right)=1$ to be different from the (4.5) one).

Definition 4.1. An almost paracontact metric Finsler structure $(\phi, \eta, \xi, G)$ is called a paracontact metric Finsler structure if

$$
d \eta^{H}(X, Y)=\Phi\left(X^{H}, Y^{H}\right), \quad d \eta^{V}(X, Y)=\Phi\left(X^{V}, Y^{V}\right) .
$$

By (4.4) and (4.6), it follows that $d \eta(X, Y)=G(X, \phi Y)$. Then we get the following

$$
d \eta\left(X^{H}, Y^{H}\right)=G\left(X^{H}, \phi Y^{H}\right)=G^{H}(X, \phi Y)=d \eta^{H}(X, Y) .
$$

Similarly, we obtain

$$
d \eta\left(X^{V}, Y^{V}\right)=d \eta^{V}(X, Y) \text { and } d \eta\left(X^{V}, Y^{H}\right)=d \eta\left(X^{H}, Y^{V}\right)=0 .
$$

Thus we deduce that $(\phi, \eta, \xi, G)$ is a paracontact metric Finsler structure if and only if the following holds

$$
\begin{aligned}
& d \eta\left(X^{H}, Y^{H}\right)=d \eta^{H}(X, Y)=G^{H}(X, \phi Y), \\
& d \eta\left(X^{V}, Y^{V}\right)=d \eta^{V}(X, Y)=G^{V}(X, \phi Y), \\
& d \eta\left(X^{H}, Y^{V}\right)=d \eta\left(X^{V}, Y^{H}\right)=0 .
\end{aligned}
$$

Moreover, if this structure is normal then it is called para-Sasakian Finsler structure.

Let $(\phi, \eta, \xi, G)$ be a paracontact metric Finsler structure on $E$. If $\xi^{H}$ and $\xi^{V}$ are Killing vector fields with respect to $G^{H}$ and $G^{V}$, respectively, then $(\phi, \eta, \xi, G)$ is called a $K$-paracontact Finsler structure on $E$ and $E$ is called a $K$-paracontact Finsler vector bundle.

Theorem 4.1. Let $(\phi, \eta, \xi, G)$ be a paracontact metric Finsler structure on E. Then $N^{(2)}=N^{(4)}=0$. Moreover, $N^{(3)}=0$ if and only if $\xi^{H}$ and $\xi^{V}$ are Killing vector fields with respect to $G^{H}$ and $G^{V}$, respectively.

Proof. Since $(\phi, \eta, \xi, G)$ is a paracontact metric Finsler structure on $E$, then we have

$$
0=G^{H}\left(\xi^{H}, \phi X^{H}\right)=d \eta^{H}\left(\xi^{H}, X^{H}\right)=\left(£_{\xi}^{H} \eta^{H}\right)\left(X^{H}\right)=N^{(4)}\left(X^{H}\right) .
$$


We also have

$$
d \eta^{H}\left(\phi X^{H}, Y^{H}\right)=G^{H}\left(\phi X^{H}, \phi Y^{H}\right)=-G^{H}\left(X^{H}, \phi^{2} Y^{H}\right)=-d \eta^{H}\left(X^{H}, \phi Y^{H}\right),
$$

which gives us $N^{(2)}\left(X^{H}, Y^{H}\right)=0$. Similarly, we obtain $N^{(2)}\left(X^{V}, Y^{V}\right)=0$. Using (4.1) and (4.6), we get

$$
d \eta^{H}\left(\phi X^{V}, Y^{H}\right)=d \eta^{H}\left(\phi Y^{H}, X^{V}\right)=d \eta^{V}\left(\phi X^{V}, Y^{H}\right)=d \eta^{V}\left(\phi Y^{H}, X^{V}\right)=0 .
$$

The above equations gives us $N^{(2)}\left(X^{V}, Y^{H}\right)=0$.

Now, we prove the second part of the Theorem. According to

$$
£_{\xi}^{H} d \eta^{H}=i_{\xi}^{H}\left(d^{2} \eta^{H}\right)+d \circ i_{\xi}^{H} d \eta^{H}=d \circ i_{\xi}^{H} d \eta^{H},
$$

Since $N^{(4)}=0$, then we obtain

$$
\left(i_{\xi^{H}} d \eta^{H}\right)\left(X^{H}\right)=d \eta^{H}\left(\xi^{H}, X^{H}\right)=N^{(4)}\left(X^{H}\right)=0 .
$$

By assumption, we have

$$
d \eta^{H}\left(\xi^{H}, X^{V}\right)=G^{H}\left(\xi^{H}, \phi X^{V}\right)=0 .
$$

By (4.8), it follows that

$$
\left(i_{\xi^{H}} d \eta^{H}\right)\left(X^{V}\right)=d \eta^{H}\left(\xi^{H}, X^{V}\right)=0 .
$$

Then (4.7) and (4.9) imply that $i_{\xi^{H}} d \eta^{H}=0$ and consequently $£_{\xi}^{H} d \eta^{H}=0$. Similarly, we obtain $£_{\xi}^{V} d \eta^{V}=0$. Therefore, we get

$$
\begin{aligned}
& 0=\left(£_{\xi}^{H} d \eta^{H}\right)\left(X, Y^{H}\right)=\left(£_{\xi}^{H} G^{H}\right)\left(X, \phi Y^{H}\right)+G^{H}\left(X,\left(£_{\xi}^{H} \phi\right)\left(Y^{H}\right)\right), \\
& 0=\left(£_{\xi}^{H} d \eta^{H}\right)\left(X, Y^{V}\right)=\left(£_{\xi}^{H} G^{H}\right)\left(X, \phi Y^{V}\right)+G^{H}\left(X,\left(£_{\xi}^{H} \phi\right)\left(Y^{V}\right)\right), \\
& 0=\left(£_{\xi}^{V} d \eta^{V}\right)\left(X, Y^{H}\right)=\left(£_{\xi}^{V} G^{V}\right)\left(X, \phi Y^{H}\right)+G^{V}\left(X,\left(£_{\xi}^{V} \phi\right)\left(Y^{H}\right)\right), \\
& 0=\left(£_{\xi}^{V} d \eta^{V}\right)\left(X, Y^{V}\right)=\left(£_{\xi}^{V} G^{V}\right)\left(X, \phi Y^{V}\right)+G^{V}\left(X,\left(£_{\xi}^{V} \phi\right)\left(Y^{V}\right)\right) .
\end{aligned}
$$

By these equations, we conclude that if $£_{\xi}^{H} G^{H}=£_{\xi}^{V} G^{V}=0$, then $N^{(3)}=0$.

Conversely, let $N^{(3)}=0$. Then from (4.10)-(4.13) we get

(i) $\left(£_{\xi}^{H} G^{H}\right)(X, \phi Y)=0, \quad(i i)\left(£_{\xi}^{V} G^{V}\right)(X, \phi Y)=0$. 
Now, we show that $\left(£_{\xi}^{H} G^{H}\right)(X, Y)=0$. It is easy to see that

$$
\left(£_{\xi}^{H} G^{H}\right)\left(X^{V}, Y^{V}\right)=0 .
$$

Using part (i) of (4.14), we obtain

$$
\begin{aligned}
\left(£_{\xi}^{H} G^{H}\right)\left(X^{H}, Y^{H}\right) & =\left(£_{\xi}^{H} G^{H}\right)\left(X^{H}, \phi^{2} Y^{H}\right)+\eta^{H}\left(Y^{H}\right)\left(£_{\xi}^{H} G^{H}\right)\left(X^{H}, \xi^{H}\right) \\
& =\eta^{H}\left(Y^{H}\right)\left(£_{\xi}^{H} G^{H}\right)\left(X^{H}, \xi^{H}\right) .
\end{aligned}
$$

Since $N^{(4)}=0$, then we have

$$
\left(£_{\xi}^{H} G^{H}\right)\left(X^{H}, \xi^{H}\right)=\left(£_{\xi}^{H} \eta^{H}\right)\left(X^{H}\right)=0 .
$$

The relations (4.15) and (4.15) give us

$$
\left(£_{\xi}^{H} G^{H}\right)\left(X^{H}, Y^{H}\right)=0 .
$$

By part (i) of (4.14), we get

$$
\begin{aligned}
\left(£_{\xi}^{H} G^{H}\right)\left(X^{H}, Y^{V}\right) & =\left(£_{\xi}^{H} G^{H}\right)\left(X^{H}, \phi^{2} Y^{V}\right)+\eta^{V}\left(Y^{V}\right)\left(£_{\xi}^{H} G^{H}\right)\left(X^{H}, \xi^{V}\right) \\
& =-\eta^{V}\left(Y^{V}\right) G^{H}\left(X^{H},\left[\xi^{H}, \xi^{V}\right]\right) .
\end{aligned}
$$

Again, using part (i) of (4.14), it follows that

$$
\begin{aligned}
0 & =\left(£_{\xi}^{H} G^{H}\right)\left(\xi^{V}, \phi^{2} Y^{H}\right)=-G^{H}\left(\left[\xi^{H}, \xi^{V}\right], \phi^{2} Y^{H}\right) \\
& =-G^{H}\left(\left[\xi^{H}, \xi^{V}\right], Y^{H}\right)+\eta^{H}\left(Y^{H}\right) G^{H}\left(\left[\xi^{H}, \xi^{V}\right], \xi^{H}\right) \\
& =-G^{H}\left(\left[\xi^{H}, \xi^{V}\right], Y^{H}\right)+\eta^{H}\left(Y^{H}\right) \eta^{H}\left(\left[\xi^{H}, \xi^{V}\right]\right) .
\end{aligned}
$$

Since $N^{(4)}=0$, then we have

$$
0=\left(£_{\xi^{V}} \eta^{H}\right)\left(\xi^{H}\right)=-\eta^{H}\left(\left[\xi^{V}, \xi^{H}\right]\right) .
$$

Plugging (4.16) in (4.16) implies that

$$
G^{H}\left(\left[\xi^{H}, \xi^{V}\right], Y^{H}\right)=0 .
$$

Then (4.16) reduces to the following

$$
\left(£_{\xi}^{H} G^{H}\right)\left(X^{H}, Y^{V}\right)=0 .
$$

It follows that $\left(£_{\xi}^{H} G^{H}\right)(X, Y)=0$, where $X, Y \in \chi(E)$. Similarly, we can obtain $\left(£_{\xi}^{V} G^{V}\right)(X, Y)=0$. This completes the proof.

Remark 4.2. In [5], the authors used the equivalence between the Killing property of $\xi$ and the Killing properties of $\xi^{H}$ and $\xi^{V}$ several times (see Lemma 5.1 and Corollary 5.2 of [5]). But it is not true. Indeed, if $\xi$ is Killing then $\xi^{H}$ and $\xi^{V}$ are not Killing, necessarily. 
In the next proposition, we explain an important relation as a big widget for our next purposes.

Proposition 4.1. Let $(\phi, \eta, \xi, G)$ be an almost paracontact metric Finsler structure on $E$. Then the following hold

$$
\begin{gathered}
2 G\left(\left(D_{X^{H}} \phi\right) Y^{H}, Z^{H}\right)=-d \Phi\left(X^{H}, \phi Y^{H}, \phi Z^{H}\right)-d \Phi\left(X^{H}, Y^{H}, Z^{H}\right) \\
-G\left(N^{(1)}\left(Y^{H}, Z^{H}\right), \phi X^{H}\right)+N^{(2)}\left(Y^{H}, Z^{H}\right) \eta\left(X^{H}\right) \\
+d \eta^{H}\left(\phi Y^{H}, X^{H}\right) \eta\left(Z^{H}\right)-d \eta^{H}\left(\phi Z^{H}, X^{H}\right) \eta\left(Y^{H}\right), \\
2 G\left(\left(D_{X^{V}} \phi\right) Y^{V}, Z^{V}\right)=-d \Phi\left(X^{V}, \phi Y^{V}, \phi Z^{V}\right)-d \Phi\left(X^{V}, Y^{V}, Z^{V}\right) \\
-G\left(N^{(1)}\left(Y^{V}, Z^{V}\right), \phi X^{V}\right)+N^{(2)}\left(Y^{V}, Z^{V}\right) \eta\left(X^{V}\right) \\
+d \eta^{V}\left(\phi Y^{V}, X^{V}\right) \eta\left(Z^{V}\right)-d \eta^{V}\left(\phi Z^{V}, X^{V}\right) \eta\left(Y^{V}\right) .
\end{gathered}
$$

Proof. By a simple calculation, we get

$$
\begin{aligned}
d \Phi\left(X^{H}, \phi Y^{H}, \phi Z^{H}\right)= & -X^{H}\left(\Phi\left(Y^{H}, Z^{H}\right)\right)-\phi Y^{H}\left(g\left(Z^{H}, X^{H}\right)\right) \\
& +\phi Y^{H}\left(\eta\left(Z^{H}\right) \eta\left(X^{H}\right)\right)+\phi Z^{H}\left(G\left(X^{H}, Y^{H}\right)\right) \\
& -\phi Z^{H}\left(\eta\left(X^{H}\right) \eta\left(Y^{H}\right)\right)-G\left(\left[X^{H}, \phi Y^{H}\right], Z^{H}\right) \\
& +\eta^{H}\left(\left[X^{H}, \phi Y^{H}\right]\right) \eta\left(Z^{H}\right)-G\left(\left[\phi Z^{H}, X^{H}\right], Y^{H}\right) \\
& +\eta^{H}\left(\left[\phi Z^{H}, X^{H}\right] \eta\left(Y^{H}\right)-\Phi\left(\left[\phi Y^{H}, \phi Z^{H}\right], X^{H}\right) .\right.
\end{aligned}
$$

Also we have

$$
\begin{aligned}
G\left(N^{(1)}\left(Y^{H}, Z^{H}\right), \phi X^{H}\right)= & \Phi\left(\left[Y^{H}, Z^{H}\right], X^{H}\right)+\Phi\left(\left[\phi Y^{H}, \phi Z^{H}\right], X^{H}\right) \\
& +G\left(\left[\phi Y^{H}, Z^{H}\right], X^{H}\right)-\eta^{H}\left(\left[\phi Y^{H}, Z^{H}\right]\right) \eta\left(X^{H}\right) \\
& +G\left(\left[Y^{H}, \phi Z^{H}\right], X^{H}\right)-\eta^{H}\left(\left[Y^{H}, \phi Z^{H}\right]\right) \eta\left(X^{H}\right) .
\end{aligned}
$$

Moreover, the following holds

$$
\begin{aligned}
& d \eta^{H}\left(\phi Y^{H}, X^{H}\right) \eta\left(Z^{H}\right)=\phi Y^{H}\left(\eta\left(X^{H}\right)\right) \eta\left(Z^{H}\right)-\eta^{H}\left(\left[\phi Y^{H}, X^{H}\right]\right) \eta\left(Z^{H}\right), \\
& d \eta^{H}\left(\phi Z^{H}, X^{H}\right) \eta\left(Y^{H}\right)=\phi Z^{H}\left(\eta\left(X^{H}\right)\right) \eta\left(Y^{H}\right)-\eta^{H}\left(\left[\phi Z^{H}, X^{H}\right]\right) \eta\left(Y^{H}\right) .
\end{aligned}
$$

If we denote the right-hand side of (4.17) by $I$, then using the above equations we can obtain the following

$$
\begin{aligned}
I= & \phi Y^{H}\left(G\left(Z^{H}, X^{H}\right)\right)-\phi Z^{H}\left(G\left(X^{H}, Y^{H}\right)\right)+G\left(\left[X^{H}, \phi Y^{H}\right], Z^{H}\right) \\
& +G\left(\left[\phi Z^{H}, X^{H}\right], Y^{H}\right)-Y^{H}\left(\Phi\left(Z^{H}, X^{H}\right)\right)-Z^{H}\left(\Phi\left(X^{H}, Y^{H}\right)\right) \\
& +\Phi\left(\left[X^{H}, Y^{H}\right], Z^{H}\right)+\Phi\left(\left[Z^{H}, X^{H}\right], Y^{H}\right)-G\left(\left[\phi Y^{H}, Z^{H}\right], X^{H}\right) \\
& -G\left(\left[Y^{H}, \phi Z^{H}\right], X^{H}\right) .
\end{aligned}
$$


Since $D$ is a Finsler connection, then it is $G$-compatible and its $(h) h$-torsion vanishes. Thus (4.17) reduces to following

$$
I=G\left(\left(\nabla_{X^{H}} \phi\right) Y^{H}, Z^{H}\right)-G\left(\nabla_{X^{H}} Z^{H}, \phi Y^{H}\right)-G\left(\nabla_{X^{H}} \phi Z^{H}, Y^{H}\right) .
$$

On the other hand, we have

$$
X^{H} G\left(Z^{H}, \phi Y^{H}\right)=G\left(\nabla_{X^{H}} Z^{H}, \phi Y^{H}\right)+G\left(Z^{H}, \nabla_{X^{H}} \phi Y^{H}\right),
$$

Since $G\left(Z^{H}, \phi Y^{H}\right)=G\left(\phi Z^{H}, Y^{H}\right)$, then by (4.19) and (4.20) we get

$$
G\left(\nabla_{X^{H}} Z^{H}, \phi Y^{H}\right)+G\left(\nabla_{X^{H}} \phi Z^{H}, Y^{H}\right)=-G\left(\left(\nabla_{X^{H}} \phi\right) Y^{H}, Z^{H}\right) .
$$

Plugging (4.21) in (4.18) give us (4.17). Similarly, we can obtain (4.17).

Proposition 4.2. Let $(\phi, \eta, \xi, G)$ be a paracontact metric Finsler structure on E. Then the following holds

$$
\begin{aligned}
2 G\left(\left(D_{X^{H}} \phi\right) Y^{H}, Z^{H}\right)= & -G\left(N^{(1)}\left(Y^{H}, Z^{H}\right), \phi X^{H}\right)+d \eta^{H}\left(\phi Y^{H}, X^{H}\right) \eta\left(Z^{H}\right) \\
& -d \eta^{H}\left(\phi Z^{H}, X^{H}\right) \eta\left(Y^{H}\right), \\
2 G\left(\left(D_{X^{V}} \phi\right) Y^{V}, Z^{V}\right)= & -G\left(N^{(1)}\left(Y^{V}, Z^{V}\right), \phi X^{V}\right)+d \eta^{V}\left(\phi Y^{V}, X^{V}\right) \eta\left(Z^{V}\right) \\
& -d \eta^{V}\left(\phi Z^{V}, X^{V}\right) \eta\left(Y^{V}\right) .
\end{aligned}
$$

Moreover, we get $D_{\xi} \phi=0$.

Proof. By Proposition 4.1, we can get (4.22), (4.22). Thus we prove $D_{\xi} \phi=0$. By $N^{(2)}=0$, we obtain $d \eta^{H}\left(\phi X^{H}, \xi^{H}\right)=0$. So plugging $X=\xi^{H}$ in (4.22) we get the following

$$
G\left(\left(D_{\xi^{H}} \phi\right) Y^{H}, Z^{H}\right)=0,
$$

which gives us

$$
G^{H}\left(\left(D_{\xi^{H}} \phi\right) Y^{H}, Z\right)=0 .
$$

We also have $G^{H}\left(\left(D_{\xi^{H}} \phi\right) Y^{V}, Z\right)=0$. Therefore, we obtain

$$
G^{H}\left(\left(D_{\xi^{H}} \phi\right) Y, Z\right)=0 .
$$

It means that $D_{\xi^{H}} \phi=0$. Similarly, we get $D_{\xi^{V}} \phi=0$. Therefore, $D_{\xi} \phi=0$. 
Using Theorem 4.1, we conclude the following.

Theorem 4.2. Let $(\phi, \eta, \xi, G)$ is a paracontact metric Finsler structure on E. Then this structure is a K-paracontact structure if and only if $N^{(3)}=0$.

Since a para-Sasakian Finsler structure is normal, then we have $N^{(3)}=0$. Thus from the above proposition we deduce the following.

Corollary 4.1. Any para-Sasakian structure on $E$ is a K-paracontact structure.

Now, we are going to find some conditions under which a paracontact metric Finsler structure on a vector bundle $E$ reduces to a K-paracontact Finsler structure. More precisely, we prove the following theorem.

Theorem 4.3. Let $(\phi, \eta, \xi, G)$ be a paracontact metric Finsler structure on E. Then this structure is a K-paracontact Finsler structure if and only if

$$
\left\{\begin{aligned}
\text { (i) } D_{X}^{H} \xi^{H}=-\frac{1}{2} \phi X^{H}, & \text { (ii) } G^{H}\left(\left[\xi^{H}, X^{V}\right]^{H}, Y^{H}\right)=0 \\
\text { (iii) } D_{X}^{V} \xi^{V}=-\frac{1}{2} \phi X^{V}, & \text { (iv) } G^{V}\left(\left[\xi^{V}, X^{H}\right]^{V}, Y^{V}\right)=0
\end{aligned}\right.
$$

Proof. Let $(\phi, \eta, \xi, G)$ be a K-paracontact Finsler structure. Then the following holds

$$
£_{\xi}^{H} G^{H}=£_{\xi}^{V} G^{V}=0 .
$$

We have

$$
\begin{aligned}
& 0=\left(£_{\xi}^{H} G^{H}\right)\left(X^{V}, Y^{H}\right)=-G^{H}\left(\left[\xi^{H}, X^{V}\right]^{H}, Y^{H}\right), \\
& 0=\left(£_{\xi}^{V} G^{V}\right)\left(X^{H}, Y^{V}\right)=-G^{V}\left(\left[\xi^{V}, X^{H}\right]^{V}, Y^{V}\right),
\end{aligned}
$$

which gives us (ii) and (iv) of (4.23).

It is easy to see that, the following holds

$$
\left(£_{\xi}^{H} G\right)\left(X^{H}, Y^{H}\right)=\left(£_{\xi}^{H} G^{H}\right)\left(X^{H}, Y^{H}\right) .
$$

Therefore

$$
\begin{aligned}
0 & =\left(£_{\xi}^{H} G\right)\left(X^{H}, Y^{H}\right)=£_{\xi}^{H} G\left(X^{H}, Y^{H}\right)-G\left(£_{\xi}^{H} X^{H}, Y^{H}\right)-G\left(X^{H}, £_{\xi}^{H} Y^{H}\right) \\
& =£_{\xi}^{H} G\left(X^{H}, Y^{H}\right)-G\left(\left[\xi^{H}, X^{H}\right]^{H}, Y^{H}\right)-G\left(X^{H},\left[\xi^{H}, Y^{H}\right]^{H}\right) .
\end{aligned}
$$

Since $D$ is symmetric, then we have

$$
\left[\xi^{H}, X^{H}\right]^{H}=D_{\xi}^{H} X^{H}-D_{X}^{H} \xi^{H} .
$$

Plugging (4.24) in (4.24) yields

$$
0=\left(D_{\xi}^{H} G\right)\left(X^{H}, Y^{H}\right)+G\left(D_{X}^{H} \xi^{H}, Y^{H}\right)+G\left(X^{H}, D_{Y}^{H} \xi^{H}\right) .
$$


Since $D$ is $G$-compatible, then $D_{\xi}^{H} G=0$. Thus

$$
G\left(D_{X}^{H} \xi^{H}, Y^{H}\right)=-G\left(X^{H}, D_{Y}^{H} \xi^{H}\right) .
$$

Similarly, we get

$$
G\left(D_{X}^{V} \xi^{V}, Y^{V}\right)=-G\left(X^{V}, D_{Y}^{V} \xi^{V}\right)
$$

Using (2.4), we obtain

$$
2 G\left(D_{X}^{H} \xi^{H}, Y^{H}\right)-2 G\left(X^{H}, D_{Y}^{H} \xi^{H}\right)=2 d \eta\left(X^{H}, Y^{H}\right) .
$$

By (4.25) and (4.27) we have

$$
2 G\left(D_{X}^{H} \xi^{H}, Y^{H}\right)-2 G\left(X^{H}, D_{Y}^{H} \xi^{H}\right)=4 G\left(D_{X}^{H} \xi^{H}, Y^{H}\right) .
$$

(4.27) and (4.28) give us

$$
2 G\left(D_{X}^{H} \xi^{H}, Y^{H}\right)=d \eta\left(X^{H}, Y^{H}\right)=G\left(X^{H}, \phi Y^{H}\right)=-G\left(\phi X^{H}, Y^{H}\right) .
$$

Hence

$$
D_{X}^{H} \xi^{H}=-\frac{1}{2} \phi X^{H}
$$

Similarly, using (4.26) we can deduce that $D_{X}^{V} \xi^{V}=-\frac{1}{2} \phi X^{V}$.

Conversely, suppose that (4.23) holds. Then from part (i) of (4.23) we have

$$
\begin{aligned}
0 & =\left(£_{\xi}^{H} G^{H}\right)\left(X^{H}, Y^{H}\right) \\
& =G\left(D_{X}^{H} \xi^{H}, Y^{H}\right)+G\left(X^{H}, D_{Y}^{H} \xi^{H}\right) \\
& =-\frac{1}{2}\left[G\left(\phi X^{H}, Y^{H}\right)+G\left(X^{H}, \phi Y^{H}\right)\right]=0 .
\end{aligned}
$$

Also (ii) gives us

$$
\left(£_{\xi}^{H} G^{H}\right)\left(X^{V}, Y^{H}\right)=0 .
$$

Therefore, considering

$$
\left(£_{\xi}^{H} G^{H}\right)\left(X^{V}, Y^{V}\right)=0,
$$

we deduce $£_{\xi}^{H} G^{H}=0$. By a similar method, we can obtain $£_{\xi}^{V} G^{V}=0$. This completes the proof.

Lemma 4.1. Let $(\phi, \eta, \xi, G)$ be a K-paracontact Finsler structure on a vector bundle $E$. Then the following holds

$$
\begin{gathered}
R\left(X^{V}, \xi^{V}\right) \xi^{V}=-\frac{1}{4}\left(X^{V}-\eta^{V}\left(X^{V}\right) \xi^{V}\right) \\
R\left(X^{H}, \xi^{H}\right) \xi^{H}=-\frac{1}{4}\left(X^{H}-\eta^{H}\left(X^{H}\right) \xi^{H}\right)-D_{\left[X^{H}, \xi^{H}\right]^{V}} \xi^{H} .
\end{gathered}
$$


Proof. Using $\left[X^{V}, \xi^{V}\right]^{H}=0, D_{\xi} \phi=0$ and (2.6), we obtain

$$
\begin{aligned}
R\left(X^{V}, \xi^{V}\right) \xi^{V} & =\frac{1}{2} \phi\left(D_{\xi^{V}} X^{V}+\left[X^{V}, \xi^{V}\right]^{V}\right) \\
& =-\frac{1}{4} \phi^{2}\left(X^{V}\right) \\
& =-\frac{1}{4}\left[X^{V}-\eta^{V}\left(X^{V}\right) \xi^{V}\right] .
\end{aligned}
$$

Similarly, we have

$$
\begin{aligned}
R\left(X^{H}, \xi^{H}\right) \xi^{H} & =\frac{1}{2} \phi\left(D_{\xi^{H}} X^{H}+\left[X^{H}, \xi^{H}\right]^{H}\right)-D_{\left[X^{H}, \xi^{H}\right]^{V}} \xi^{H} \\
& =-\frac{1}{4} \phi^{2}\left(X^{H}\right)-D_{\left[X^{H}, \xi^{H}\right]^{V}} \xi^{H} \\
& =-\frac{1}{4}\left(X^{H}-\eta^{H}\left(X^{H}\right) \xi^{H}\right)-D_{\left[X^{H}, \xi^{H}\right]^{V}} \xi^{H}
\end{aligned}
$$

This completes the proof.

Theorem 4.4. Let $(\phi, \eta, \xi, G)$ be a K-paracontact Finsler structure on E. Then the following holds

(i) the vertical flag curvature of all plane sections containing $\xi^{V}$ is equal to $-\frac{1}{4}$;

(ii) the horizontal flag curvature of all plane sections containing $\xi^{H}$ is equal to $-\frac{1}{4}$ if and only if $G\left(D_{\left[X^{H}, \xi^{H}\right]}^{V} \xi^{H}, X^{H}\right)=0$.

Proof. Let $X^{V}$ be a unit vector field orthogonal to $\xi^{V}$. Then

$$
\eta^{V}\left(X^{V}\right)=0 .
$$

Consequently, (4.29) gives us

$$
R\left(X^{V}, \xi^{V}\right) \xi^{V}=-\frac{1}{4} X^{V}
$$

Therefore, we get

$$
K\left(X^{V}, \xi^{V}\right)=G^{V}\left(R\left(X^{V}, \xi^{V}\right) \xi^{V}, X^{V}\right)=-\frac{1}{4} G\left(X^{V}, X^{V}\right)=-\frac{1}{4} .
$$

Similarly, if $X^{H}$ is a unit vector field orthogonal to $\xi^{H}$, then from (4.30) we get

$$
\begin{aligned}
K\left(X^{H}, \xi^{H}\right) & =G^{H}\left(R\left(X^{H}, \xi^{H}\right) \xi^{H}, X^{H}\right) \\
& =-\frac{1}{4} G\left(X^{H}, X^{H}\right)-G\left(D_{\left[X^{H}, \xi^{H}\right]^{V}} \xi^{H}, X^{H}\right) \\
& =-\frac{1}{4}-G\left(D_{\left[X^{H}, \xi^{H}\right]^{V}} \xi^{H}, X^{H}\right) .
\end{aligned}
$$

Therefore $K\left(X^{H}, \xi^{H}\right)=-\frac{1}{4}$ holds if and only if $G\left(D_{\left[X^{H}, \xi^{H}\right]^{V}} \xi^{H}, X^{H}\right)=0$. 
Remark 4.3. In Theorem 6.1 of [5] and Theorem 4.2 from [19], the authors consider only $G^{H}\left(R\left(X^{H}, \xi^{H}\right) \xi^{H}, X^{H}\right)$ and $G^{V}\left(R\left(X^{V}, \xi^{V}\right) \xi^{V}, X^{V}\right)$ to compute the flag curvature of a plane which contains $\xi$. But they forgot some terms such as $G^{H}\left(R\left(X^{V}, \xi^{H}\right) \xi^{H}, X^{H}\right)$ and $G^{H}\left(R\left(X^{V}, \xi^{V}\right) \xi^{H}, X^{H}\right)$ in computing the flag curvature. Indeed, they computed only vertical and horizontal flag curvatures.

Now, we are going to study some properties of the para-Sasakian Finsler structure on a vector bundle. First, we prove the following.

Theorem 4.5. Let $(\phi, \eta, \xi, G)$ be a para-Sasakian Finsler structure on a vector bundle $E$. Then the following relations hold

$$
\begin{aligned}
\left(D_{X}^{H} \phi\right) Y^{H} & =\frac{1}{2}\left\{\eta^{H}\left(Y^{H}\right) X^{H}-G^{H}\left(X^{H}, Y^{H}\right) \xi^{H}\right\}, \\
\left(D_{X}^{V} \phi\right) Y^{V} & =\frac{1}{2}\left\{\eta^{V}\left(Y^{V}\right) X^{V}-G^{V}\left(X^{V}, Y^{V}\right) \xi^{V}\right\} .
\end{aligned}
$$

Moreover, the Riemannian curvature satisfies the following

$$
\begin{gathered}
R\left(X^{V}, Y^{V}\right) \xi^{V}=\frac{1}{4}\left\{\eta^{V}\left(X^{V}\right) Y^{V}-\eta^{V}\left(Y^{V}\right) X^{V}\right\}, \\
R\left(X^{H}, Y^{H}\right) \xi^{H}=\frac{1}{4}\left\{\eta^{H}\left(X^{H}\right) Y^{H}-\eta^{H}\left(Y^{H}\right) X^{H}\right\}-D_{\left[X^{H}, Y^{H}\right]}^{V} \xi^{H} .
\end{gathered}
$$

Proof. Since $(\phi, \eta, \xi, G)$ is a para-Sasakian Finsler structure, then $\Phi=d \eta$ and $N^{(1)}=N^{(2)}=0$. Thus by (4.17), we obtain

$$
\begin{aligned}
2 G\left(\left(D_{X}^{H} \phi\right) Y^{H}, Z^{H}\right) & =d \eta^{H}\left(\phi Y^{H}, X^{H}\right) \eta\left(Z^{H}\right)-d \eta^{H}\left(\phi Z^{H}, X^{H}\right) \eta\left(Y^{H}\right) \\
& =G\left(\phi Y^{H}, \phi X^{H}\right) \eta\left(Z^{H}\right)-G\left(\phi Z^{H}, \phi X^{H}\right) \eta\left(Y^{H}\right) \\
& =-G\left(X^{H}, Y^{H}\right) \eta\left(Z^{H}\right)+G\left(X^{H}, Z^{H}\right) G\left(\xi^{H}, Y^{H}\right) \\
& =G\left(\eta\left(Y^{H}\right) X^{H}-G\left(X^{H}, Y^{H}\right) \xi^{H}, Z^{H}\right) .
\end{aligned}
$$

This implies (4.31). With similar computations, one can obtain (4.32).

Using (2.6), Theorem 4.2 and Corollary 4.1, we have

$$
\begin{aligned}
R\left(X^{V}, Y^{V}\right) \xi^{V} & =D_{X}^{V} D_{Y}^{V} \xi^{V}-D_{Y}^{V} D_{X}^{V} \xi^{V}-D_{\left[X^{V}, Y^{V}\right]}^{V} \xi^{V} \\
& =D_{X}^{V}\left(-\frac{1}{2} \phi Y^{V}\right)-D_{Y}^{V}\left(-\frac{1}{2} \phi X^{V}\right)+\frac{1}{2} \phi\left[X^{V}, Y^{V}\right]^{V} \\
& =-\frac{1}{2}\left(D_{X}^{V} \phi\right) Y^{V}+\frac{1}{2}\left(D_{Y}^{V} \phi\right) X^{V} .
\end{aligned}
$$

By (4.32) and (4.35) we get

$$
R\left(X^{V}, Y^{V}\right) \xi^{V}=\frac{1}{4}\left\{\eta^{V}\left(X^{V}\right) Y^{V}-\eta^{V}\left(Y^{V}\right) X^{V}\right\}
$$


Similarly, using (4.31) we obtain

$$
\begin{aligned}
R\left(X^{H},\right. & \left.Y^{H}\right) \xi^{H}=D_{X}^{H} D_{Y}^{H} \xi^{H}-D_{Y}^{H} D_{X}^{H} \xi^{H}-D_{\left[X^{H}, Y^{H}\right]}^{H} \xi^{H}-D_{\left[X^{H}, Y^{H}\right]}^{V} \xi^{H} \\
& =D_{X}^{H}\left(-\frac{1}{2} \phi Y^{H}\right)-D_{Y}^{H}\left(-\frac{1}{2} \phi X^{H}\right)+\frac{1}{2} \phi\left[X^{H}, Y^{H}\right]^{H}-D_{\left[X^{H}, Y^{H}\right]}^{V} \xi^{H} \\
& =-\frac{1}{2}\left(D_{X}^{H} \phi\right) Y^{H}+\frac{1}{2}\left(D_{Y}^{H} \phi\right) X^{H}-D_{\left[X^{H}, Y^{H}\right]}^{V} \xi^{H} \\
& =\frac{1}{4}\left\{\eta^{H}\left(X^{H}\right) Y^{H}-\eta^{H}\left(Y^{H}\right) X^{H}\right\}-D_{\left[X^{H}, Y^{H}\right]}^{V} \xi^{H} .
\end{aligned}
$$

This completes the proof.

A plane section in $V_{u} E$ is called a vertical $\phi$-section if there exists a unit vector $X^{V}$ in $V_{u} E$ orthogonal to $\xi^{V}$ such that $\left\{X^{V}, \phi X^{V}\right\}$ span the section. The vertical flag curvature $K\left(X^{V}, \phi X^{V}\right)$ is called vertical $\phi$-flag curvature.

Proposition 4.3. Let $(\phi, \eta, \xi, G)$ be a para-Sasakian Finsler structure on E. Suppose that $E$ is locally symmetric. Then it has a vertical $\phi$-flag curvature $-\frac{1}{4}$.

Proof. Let $X^{V} \neq 0$ be a vector field on $E$ orthogonal to $\xi^{V}$. Then we have $\eta^{V}\left(X^{V}\right)=G^{V}\left(X^{V}, \eta^{V}\right)=0$. By direct conclusion we obtain

$$
\begin{aligned}
\left(D_{\phi X^{V}} R\right)\left(X^{V}, \phi X^{V}\right) \xi^{V}=\frac{1}{2} & {\left[\phi R\left(X^{V}, \phi X^{V}\right) \phi X^{V}-\frac{1}{4} G^{V}\left(X^{V}, \phi X^{V}\right) \phi^{2} X^{V}\right.} \\
& \left.+\frac{1}{4} G^{V}\left(\phi X^{V}, \phi X^{V}\right) \phi X^{V}\right] .
\end{aligned}
$$

Considering $G\left(X^{V}, \phi X^{V}\right)=-G\left(\phi X^{V}, X^{V}\right)$, we have $G\left(X^{V}, \phi X^{V}\right)=0$. Using this equation and noting that $E$ is locally symmetric (4.36) gives us

$$
\phi R\left(X^{V}, \phi X^{V}\right) \phi X^{V}+\frac{1}{4} G^{V}\left(\phi X^{V}, \phi X^{V}\right) \phi X^{V}=0 .
$$

By (4.37), we get

$$
G\left(\phi R\left(X^{V}, \phi X^{V}\right) \phi X^{V}, \phi X^{V}\right)+\frac{1}{4} G\left(\phi X^{V}, \phi X^{V}\right) G\left(\phi X^{V}, \phi X^{V}\right)=0 .
$$

Since $\eta^{V}\left(X^{V}\right)=0$, then (4.38) gives us

$$
G\left(R\left(X^{V}, \phi X^{V}\right) \phi X^{V}, X^{V}\right)=\frac{1}{4} G^{2}\left(\phi X^{V}, \phi X^{V}\right) .
$$

Therefore, we obtain

$$
K\left(X^{V}, \phi X^{V}\right)=\frac{G\left(R\left(X^{V}, \phi X^{V}\right) \phi X^{V}, X^{V}\right)}{G\left(X^{V}, X^{V}\right) G\left(\phi X^{V}, \phi X^{V}\right)}=-\frac{1}{4} .
$$

It means that $E$ has a vertical $\phi$-flag curvature $-\frac{1}{4}$. 


\subsection{Horizontal and Vertical Ricci Tensors}

The horizontal Ricci tensor $S^{H}$ of an $(n+m)$-dimensional para-Sasakian Finsler manifold $E$ is given by

$$
\begin{aligned}
S^{H}\left(X^{H}, Y^{H}\right) & =\sum_{i=1}^{n-1} G\left(R\left(X^{H}, E_{i}^{H}\right) E_{i}^{H}, Y^{H}\right)+G\left(R\left(X^{H}, \xi^{H}\right) \xi^{H}, Y^{H}\right) \\
& =\sum_{i=1}^{n-1} G\left(R\left(E_{i}^{H}, X^{H}\right) Y^{H}, E_{i}^{H}\right)+G\left(R\left(\xi^{H}, X^{H}\right) Y^{H}, \xi^{H}\right),
\end{aligned}
$$

where $\left\{E_{1}^{H}, E_{2}^{H}, \ldots, E_{n-1}^{H}, \xi^{H}\right\}$ is a local orthonormal frame of $H_{u} E$. Similarly, the vertical Ricci tensor of an $(n+m)$-dimensional para-Sasakian Finsler manifold $E$ is given by

$$
\begin{aligned}
S^{V}\left(X^{V}, Y^{V}\right) & =\sum_{i=1}^{m-1} G\left(R\left(X^{V}, E_{i}^{V}\right) E_{i}^{V}, Y^{V}\right)+G\left(R\left(X^{V}, \xi^{V}\right) \xi^{V}, Y^{V}\right) \\
& =\sum_{i=1}^{m-1} G\left(R\left(E_{i}^{V}, X^{V}\right) Y^{V}, E_{i}^{V}\right)+G\left(R\left(\xi^{V}, X^{V}\right) Y^{V}, \xi^{V}\right),
\end{aligned}
$$

where $\left\{E_{1}^{V}, E_{2}^{V}, \ldots, E_{m-1}^{V}, \xi^{V}\right\}$ is a local orthonormal frame of $V_{u} E$.

Proposition 4.4. The horizontal and vertical Ricci tensors $S^{H}$ and $S^{V}$ of a $(n+$ $m$ )-dimensional para-Sasakian Finsler manifold satisfy the following equations:

$$
\left\{\begin{array}{l}
\text { (i) } S^{H}\left(X^{H}, \xi^{H}\right)=\frac{1-n}{4} \eta^{H}\left(X^{H}\right)-\sum_{i=1}^{n-1} G\left(D_{\left[E_{i}^{H}, X^{H}\right]}^{V} \xi^{H}, E_{i}^{H}\right) \\
(i i) S^{V}\left(X^{V}, \xi^{V}\right)=\frac{1-m}{4} \eta^{V}\left(X^{V}\right) \\
(i i i) S^{H}\left(\xi^{H}, \xi^{H}\right)=\frac{1-n}{4}-\sum_{i=1}^{n-1} G\left(D_{\left[E_{i}^{H}, \xi^{H}\right]}^{V} \xi^{H}, E_{i}^{H}\right) \\
\text { (iv) } S^{V}\left(\xi^{V}, \xi^{V}\right)=\frac{1-m}{4}
\end{array}\right.
$$

Proof. Using (4.34) and (4.39), one can obtain the following:

$$
\begin{aligned}
S^{H}\left(X^{H}, \xi^{H}\right) & =\sum_{i=1}^{n-1} G\left(R\left(E_{i}^{H}, X^{H}\right) \xi^{H}, E_{i}^{H}\right) \\
= & \sum_{i=1}^{n-1} G\left(\frac{1}{4} \eta^{H}\left(E_{i}^{H}\right) X^{H}-\frac{1}{4} \eta^{H}\left(X^{H}\right) E_{i}^{H}-D_{\left[E_{i}^{H}, X^{H}\right]}^{V} \xi^{H}, E_{i}^{H}\right) .
\end{aligned}
$$

Since $E_{i}^{H}$ is orthogonal to $\xi^{H}$, then we have $\eta^{H}\left(E_{i}^{H}\right)=G\left(E_{i}^{H}, \xi^{H}\right)=0$. By (4.40) and $G\left(E_{i}^{H}, E_{i}^{H}\right)=1$, we get part (i) of (4.39). Plugging $X^{H}=\xi^{H}$ in (i) and using 
$\eta^{H}\left(X^{H}\right)=1$ implies (iii). Similarly, (4.33) and (4.39) give us

$$
\begin{aligned}
S^{V}\left(X^{V}, \xi^{V}\right) & =\sum_{i=1}^{m-1} G\left(R\left(E_{i}^{V}, X^{V}\right) \xi^{V}, E_{i}^{V}\right) \\
& =\frac{1}{4} \sum_{i=1}^{m-1} G\left(\eta^{V}\left(E_{i}^{V}\right) X^{V}-\eta^{V}\left(X^{V}\right) E_{i}^{V}, E_{i}^{V}\right) \\
& =\frac{1-m}{4} \eta^{V}\left(X^{V}\right) .
\end{aligned}
$$

By setting $X^{V}=\xi^{V}$ in (4.41), we get (iv).

According to parts (i) and (iii) of (4.39), one can deduce the following easily.

Corollary 4.2. For an $(n+m)$-dimensional para-Sasakian Finsler manifold, the following holds

i) $S^{H}\left(X^{H}, \xi^{H}\right)=\frac{1-n}{4} \eta^{H}\left(X^{H}\right)$ is equivalent to vanishing of

$$
\sum_{i=1}^{n-1} G\left(D_{\left[E_{i}^{H}, X^{H}\right]}^{V} \xi^{H}, E_{i}^{H}\right)
$$

ii) $S^{H}\left(\xi^{H}, \xi^{H}\right)=\frac{1-n}{4}$ is equivalent to vanishing of

$$
\sum_{i=1}^{n-1} G\left(D_{\left[E_{i}^{H}, \xi^{H}\right]}^{V} \xi^{H}, E_{i}^{H}\right)
$$

Using Lemma 4.1, we have the following proposition.

Proposition 4.5. The horizontal and vertical Ricci tensors $S^{H}$ and $S^{V}$ of a $(n+$ $m$ )-dimensional K-paracontact Finsler vector bundle satisfy the following equations:

$$
S^{H}\left(\xi^{H}, \xi^{H}\right)=\frac{1-n}{4}-\sum_{i=1}^{n-1} G\left(D_{\left[E_{i}^{H}, \xi^{H}\right]}^{V} \xi^{H}, E_{i}^{H}\right), \quad S^{V}\left(\xi^{V}, \xi^{V}\right)=\frac{1-m}{4} .
$$

Proposition 4.5 have an easy consequence as follows.

Corollary 4.3. For a $(n+m)$-dimensional K-paracontact Finsler vector bundle $E, S^{H}\left(\xi^{H}, \xi^{H}\right)=\frac{1-n}{4}$ is equivalent to the vanishing of

$$
\sum_{i=1}^{n-1} G\left(D_{\left[E_{i}^{H}, \xi^{H}\right]}^{V} \xi^{H}, E_{i}^{H}\right)
$$




\section{REFEREN CES}

1. Bejan C. L and Druţă-RomaniuC S. L., Connections which are harmonic with respect to general natural metrics, Diff. Geom. Appl. 30(2012), 306-317.

2. Calvaruso G., Symplectic, complex and Kähler structures on four-dimensional generalized symmetric spaces, Diff. Geom. Appl. 29(2011), 758-769.

3. Ivanov S, VAssilev D and Zamkovoy Z., Conformal paracontact curvature and the local flatness theorem, Geom. Dedicata, 144(2010), 79-100.

4. KANEYUKI S and Willams FL., Almost paracontact and parahodge structures on manifolds, Nagoya Math. J. 99(1985), 173-187.

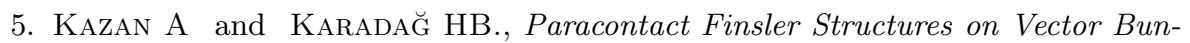
dles, British J. Math. Computer Sci., 4(24) (2014), 3403-3426.

6. Miron R, Hrimiuc D, Shimada H and Sabau SV., The Geometry of Hamilton and Lagrange Spaces, Kluwer. Acad. Pub. FTPH. 118, 2001.

7. Cappelletti-Montano B., Bi-Legendrian structures and paracontact geometry, Int. J. Geom. Methods Mod. Phys. 6(2009), 487-504.

8. Cappelletti-Montano B, Erken IK and Murathan C., Nullity conditions in paracontact geometry, Diff. Geom. Appl., 30(2012), 665-693.

9. SAsAKI S., On paracontact Riemannian manifolds, TRU Math. 16(2) (1980), $75-86$.

10. SASAKI S., On differentiable manifolds with certain structures which are closely related to almost contact structure, Tohoku. Math. J. 12(2)(1960), 459-476.

11. P SAto I., On a structure similar to the almost contact structure, Tensor (N. S), 30(1976), 219-224.

12. SinHA BB and SAI PRASAD KL., Almost paracontact semi-symmetric metric Finsler connections on vector bundle, Indian J. Pure. Appl. Math, 26(3) (1995), 249-257.

13. SinHA BB and YADAV RK., An almost contact Finsler structures on vector bundle, Indian J. Pure. Appl. Math, 19(1) (1988), 27-35.

14. SinHA BB and YADAV RK., Almost contact semi-symmetric metric Finsler connections on vector bundle, Indian J. Pure. Appl. Math, 22(1) (1991), 29-39.

15. VACARU SI and VICOL NA., Nonlinear connections and Clifford structures, arXiv: math/0205190v2 [math.DG], (2002).

16. VACARU S., Superstrings in higher order extensions of Finsler superspaces, Nucl. Phys. B, 434(1997) 590-656.

17. Zamkovoy S., Canonical connections on paracontact manifolds, Ann. Glob. Anal. Geom. 36(2009), 37-60.

18. Zamkovoy S., Canonical connections in paracontact manifolds, ArXiv: math/0707.1787v2 [math.DG], (2007).

19. Yalinis AF and ÇALIŞKan N., Sasakian Finsler manifolds, Turkish J. Math., 37 (2013), 319-339. 
e-peyghan@araku.ac.ir, esasharahi@gmail.com

Department of Mathematics

Faculty of Science,

Arak University, Arak 38156-8-8349, Iran. 